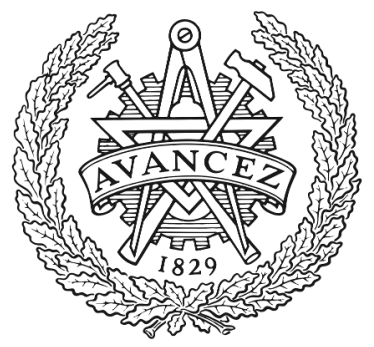

CHALMERS

UNIVERSITY OF TECHNOLOGY

\title{
A new approximation to modulation-effect analysis based on empirical mode decomposition
}

Downloaded from: https://research.chalmers.se, 2023-04-26 07:37 UTC

Citation for the original published paper (version of record):

Altintas, A., Davidson, L., Shia-Hui, P. (2019). A new approximation to modulation-effect analysis based on empirical mode decomposition. Physics of Fluids, 31(2).

http://dx.doi.org/10.1063/1.5079601

N.B. When citing this work, cite the original published paper. 


\section{A new approximation to modulation- effect analysis based on empirical mode decomposition}

Cite as: Phys. Fluids 31, 025117 (2019); https://doi.org/10.1063/1.5079601

Submitted: 01 November 2018 . Accepted: 31 January 2019 . Published Online: 22 February 2019

A. Altıntaş (D) L. Davidson (D), and S. H. Peng

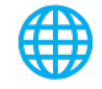

\section{ARTICLES YOU MAY BE INTERESTED IN}

Local dynamic perturbation effects on amplitude modulation in turbulent boundary layer flow based on triple decomposition

Physics of Fluids 31, 025120 (2019); https://doi.org/10.1063/1.5083224

\section{Referee acknowledgment for 2018}

Physics of Fluids 31, 020201 (2019); https://doi.org/10.1063/1.5090536

Three dimensional structure of the unsteady wake of an axisymmetric body

Physics of Fluids 31, 025113 (2019); https://doi.org/10.1063/1.5078379 


\title{
A new approximation to modulation-effect analysis based on empirical mode decomposition
}

\author{
Cite as: Phys. Fluids 31, 025117 (2019); doi: 10.1063/1.5079601 \\ Submitted: 1 November 2018 - Accepted: 31 January 2019 • \\ Published Online: 22 February 2019
}

\section{A. Altıntaş, a) (D) L. Davidson, (D) and S. H. Peng}

\begin{abstract}
AFFILIATIONS
Division of Fluid Dynamics, Department of Mechanics and Maritime Sciences, Chalmers University of Technology, SE-412 96 Gothenburg, Sweden
\end{abstract}

\begin{abstract}
a) Author to whom correspondence should be addressed: altintas@chalmers.se
b) Also at: Swedish Defence Research Agency, FOI, SE-164 90 Stockholm, Sweden.
\end{abstract}

\begin{abstract}
The modulation effect, namely, the amplification or attenuation of near-wall small-scale (SS) structures by outer large-scale (LS) structures, is one of two commonly accepted ways that outer LS turbulent fluctuations can influence near-wall ones. Mode decomposition based on filtering is widely used to analyze the modulation effect. In the present study, a new approximation is proposed based on empirical mode decomposition (EMD) to investigate the aforementioned amplitude modulation effect. Both methods are used, and their results are compared for two-point and single-point analyses. It has been shown that the LS and SS signals that are decomposed by filtering and EMD follow identical paths. Despite the similarities of the signals, the suggested method exhibits a slightly higher correlation coefficient R compared to the method based on filtering for the two-point analysis. For the one-point analysis, however, the suggested method gives a rational correlation coefficient for the one-point analysis compared to the two-point analysis, while the existing method seems far from giving a rational correlation coefficient value, which is too low compared to that of the two-point analysis. The suggested method is relevant to many recent studies that questioned the reliability of calculating the correlation coefficient with the existing method. The variation of $R$ for identical signals extends the discussion of the correlation-coefficient calculations to the very first process, namely, obtaining LS and SS data from the original signal.
\end{abstract}

Published under license by AIP Publishing. https://doi.org/10.1063/1.5079601

\section{INTRODUCTION}

Many studies have addressed the existence of large-scale (LS) and very-large-scale motions that are very important for wall turbulence at high Reynolds numbers. ${ }^{7-3}$ Jiménez $^{1}$ reported the existence of large eddies with streamwise lengths of the order of 10-20 boundary-layer thicknesses in the logarithmic region of wall-bounded flows. These large eddies involve mostly streamwise velocity fluctuations and contain most of the streamwise kinetic energy. Many subsequent studies have reported that log-law LS motions strongly influence near-wall turbulent structures. ${ }^{4-8}$

There are two commonly accepted ways that outer LS structures can affect near-wall turbulence, namely, footprinting and the modulation effect. In footprinting, LS motions interact with the corresponding large near-wall motions, resulting in footprints of the outer LS structures in the vicinity of the wall. The modulation effect is amplification or attenuation of near-wall small-scale (SS) structures by the LS motions in the log-law region. ${ }^{9}$

To investigate the modulation effect in the velocity signal, the latter must be decomposed into large and small scales. Two methods are frequently used to do this, namely, (i) proper mode decomposition and (ii) filtering by defining the cutoff wavelength that separates the large and small scales. Mathis et al. ${ }^{5}$ proposed what is now a widely used method for analyzing amplitude modulation, in which filtering is used to decompose the signal. However, a number of recent studies have criticized the reliability of the correlation coefficient defined by that method. The criticism is focused on how the statistical asymmetry of the signal affects the modulation. Schlatter and Örlü ${ }^{6}$ showed that the correlation coefficient 


$$
R=\frac{\overline{u_{L}^{+} E_{L}\left(u_{S}^{+}\right)}}{\sqrt{u_{L}^{+2}} \sqrt{E_{L}\left(u_{S}^{+}\right)^{2}}}
$$

where $E$ denotes the envelope of the signal, can be used to quantify the amplitude modulation and is related to the skewness of the signal. However, they concluded that because $R$ depends on the skewness of the original data, it is insufficient for defining the degree of modulation. Mathis et al. ${ }^{7}$ then showed that the LS motions strengthen with increasing Reynolds number and that the amplitude modulation effect helps increase the skewness. Recently, Agostini et al. ${ }^{10}$ suggested accounting for the skewness of the SS fluctuations to obtain their envelope, and they presented a new method that defines two separate envelopes for the positive and negative small-scale fluctuations.

In the present study, we use both the existing filtering method $^{5}$ and empirical mode decomposition (EMD) as a new approximation to decompose signals and analyze the amplitude modulation effect of the outer LS structures on the inner SS structures. EMD splits the raw signal into oscillatory components known as intrinsic mode functions (IMFs). ${ }^{11}$ Unlike filtering, which divides a given signal into two energy containing signals, each IMF contains different energy levels. Dynamical Mode Decomposition (DMD) is another widely used method to extract dynamic modes of flow fields. ${ }^{12} \mathrm{How}^{-}$ ever, whereas the EMD method can decompose complex, non-linear, non-stationary data to a small number of components, DMD needs modifications to improve the performance when the spatial complexity is smaller than the spectral complexity. ${ }^{13}$

However, in this study, it is shown that the SS and LS signals obtained by both decomposition methods are identical regarding the paths that they follow, but the correlation coefficient $\mathrm{R}$ obtained differs between the two methods. Therefore, the present study extends the discussion of estimating the correlation coefficient to the very first step of analyzing the modulation effect, namely, decomposing the signal into large and small scales.

\section{NUMERICAL METHODS}

An implicit, two-step time-advancement finite-volume method is used. ${ }^{14}$ Central differencing is used in the spatial domain, and the Crank-Nicolson scheme is used in the temporal domain. Discretizing the Navier-Stokes equation for $u_{i}$, it becomes

$$
u_{i}^{n+1}=u_{i}^{n}+\Delta t H\left(u_{i}^{n}, u_{i}^{n+1}\right)-\frac{1}{\rho} \alpha \Delta t \frac{\partial p^{n+1}}{\partial x_{i}}-\frac{1}{\rho}(1-\alpha) \Delta t \frac{\partial p^{n}}{\partial x_{i}},
$$

where $H\left(u_{i}^{n}, u_{i}^{n+1}\right)$ includes convection, viscosity, and source terms and $\alpha=0.5$ (Crank-Nicolson). Equation (1) is solved to give $u_{i}^{n+1}$, which does not satisfy continuity. An intermediate velocity field is computed by subtracting the implicit part of the pressure gradient,

$$
u_{i}^{*}=u_{i}^{n+1}+\frac{1}{\rho} \alpha \Delta t \frac{\partial p^{n+1}}{\partial x_{i}} .
$$

Taking the divergence of Eq. (2) while requiring continuity (for the face velocities, which are obtained by linear interpolation) be satisfied on level $n+1$, i.e., $\partial u_{i, f}^{n+1} / \partial x_{i}=0$, we obtain

$$
\frac{\partial^{2} p^{n+1}}{\partial x_{i} \partial x_{i}}=\frac{\rho}{\Delta t \alpha} \frac{\partial u_{i, f}^{*}}{\partial x_{i}} .
$$

The numerical procedure at each time step is summarized as follows: ${ }^{15}$

1. Solve the discretized filtered Navier-Stokes equation for $u, v$, and $w$.

2. Create an intermediate velocity field $u_{i}^{*}$ from Eq. (2).

3. The Poisson equation [Eq. (3)] is solved with an efficient multigrid method. ${ }^{16}$

4. Compute the face velocities (which satisfy continuity) from the pressure and the intermediate velocity as

$$
u_{i, f}^{n+1}=u_{i, f}^{*}-\frac{1}{\rho} \alpha \Delta t\left(\frac{\partial p^{n+1}}{\partial x_{i}}\right)_{f} .
$$

5. Steps 1-4 are performed until convergence is achieved (normally after two or three iterations). The convergence criteria are $10^{-7}$ for velocity and $10^{-5}$ for pressure. The residuals are computed using the $\mathrm{L} 1 \mathrm{norm}$ and are scaled with the integrated streamwise volume flux (continuity equation) and momentum flux (momentum equations).

6. Next time step.

Note that although no explicit dissipation is added to prevent odd-even decoupling, dissipation is present implicitly. The intermediate velocity field is computed at the cell centers [see Eq. (2)] subtracting a pressure gradient. When, having solved the pressure Poisson equation, the face velocity field is computed, the pressure gradient at the faces [see Eq. (4)] is added. This is very similar to the Rhie-Chow dissipation. ${ }^{17}$

A constant volumetric driving force is used in the streamwise momentum equation by which the frictional Reynolds number $\operatorname{Re}_{\tau}=550$ is prescribed based on $u_{\tau}$ (friction velocity) and $\delta$ (half the channel height). The friction velocity, $u_{\tau}$, is based on the driving pressure gradient. Periodic boundary conditions are used in the streamwise and spanwise directions, while the usual no-slip boundary conditions are enforced at the walls. With respect to the streamwise, wallnormal, and spanwise directions, the domain size is $2 \pi \delta \times 2 \delta$ $\times \pi \delta$, meshed with $258 \times 98 \times 258$ cells. The grid resolution is $\Delta x^{+} \approx 13, \Delta z^{+} \approx 6$, and a stretching of 1.03 is used in the wall-normal direction.

The time step is $\Delta t=0.0012$, and the nondimensional time step is $\Delta t^{+}=\Delta t u_{\tau}^{2} / v=0.22$, where $v$ is the kinematic viscosity. The variables $u, v$, and $w$ represent the streamwise, wallnormal, and spanwise velocities, respectively. Unless stated otherwise, the results are averaged over time and over all homogeneous directions (i.e., $x_{1}, x_{3}$, and $t$ ); the average is denoted by an overbar $(\overline{)})$.

\section{AMPLITUDE MODULATION AND HILBERT TRANSFORM}

Amplitude modulation refers to the modulation of a highfrequency signal (carrier signal) by a low-frequency component (modulating signal). Mathis et al. ${ }^{5}$ described Hilbert 


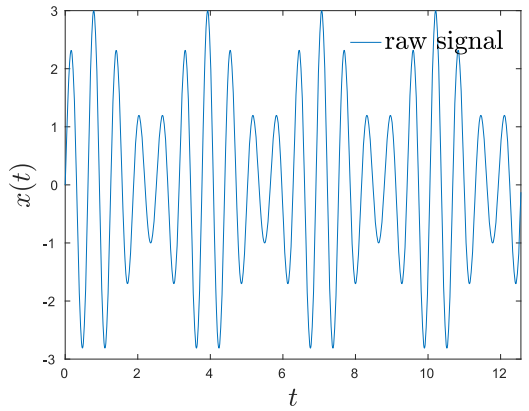

(a)

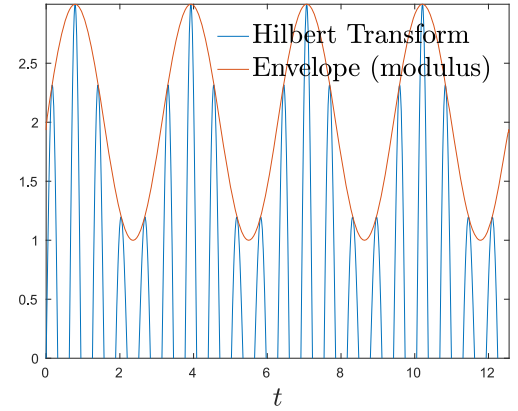

(b) transformation briefly, and we use the same simple sinusoidal function that they used to describe how Hilbert transformation is used to find an envelope to an amplitude-modulated signal.

For any amplitude-modulated signal $x(t)$, it is possible to find a modulating signal $m(t)$ by applying Hilbert transformation to the original signal to obtain a Hilbert-transformed signal $\mathscr{X}$. With the definition of the Hilbert transform, $\mathscr{X}$ and the original signal $x(t)$ form a harmonic conjugate pair, the associated complex analytic signal $Z(t)$ of $x(t)$ defined as

$$
Z(t)=x(t)+i \mathscr{X}(\mathrm{t})
$$

The modulus of this complex signal, namely,

$$
\mathrm{A}(\mathrm{t})=\sqrt{x(t)^{2}+\mathscr{X}(\mathrm{t})^{2}}
$$

gives the envelope of the raw signal $x(t)$.

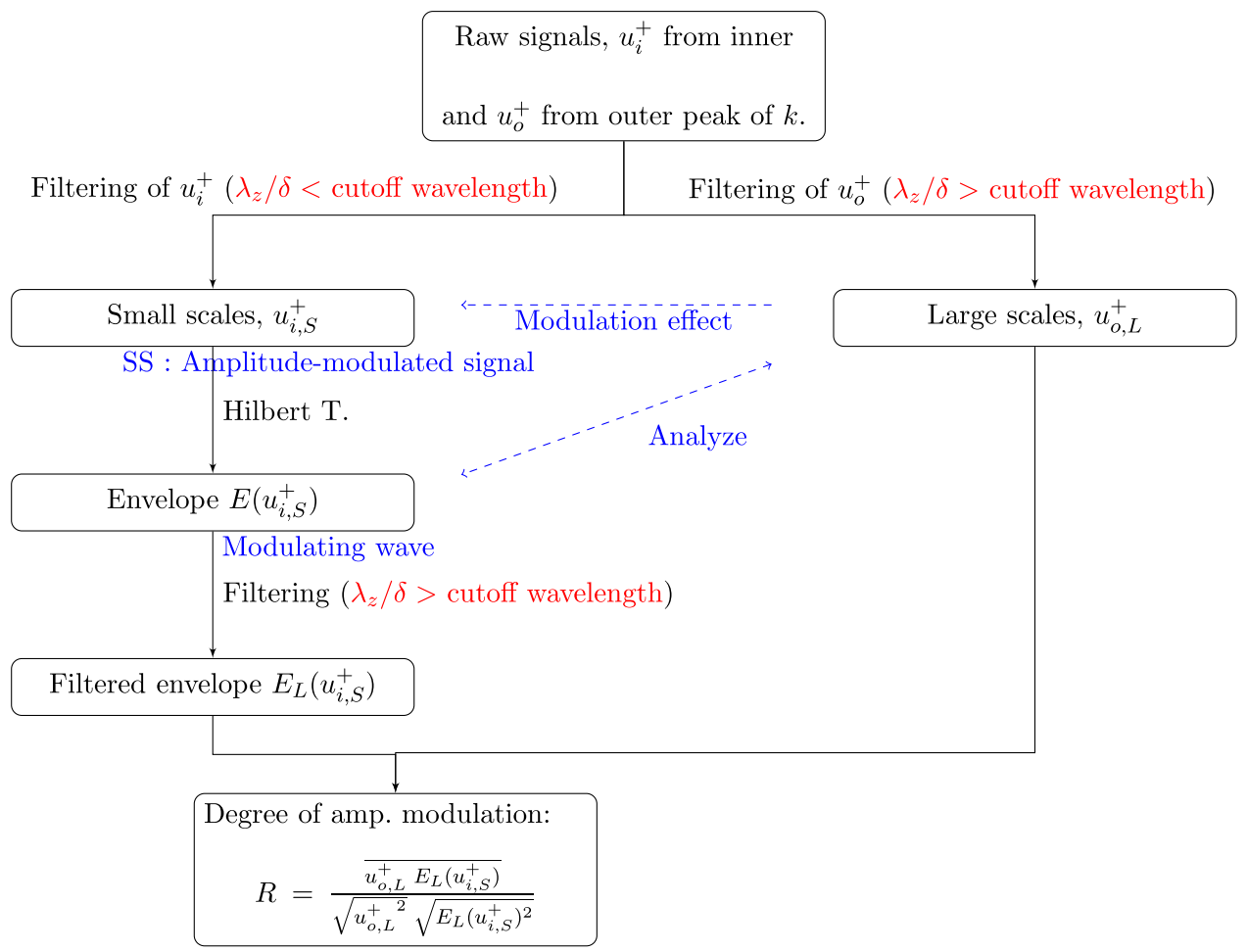

FIG. 1. Example of finding a modulating wave by Hilbert transformation: (a) raw signal. (b) Hilbert transformation is applied to give the modulating wave, shown in red.
Let $x(t)=(B+\sin (2 t)) \sin (10 t)$, where $c(t)=\sin (10 t)$ and $m(t)$ $=\sin (2 \mathrm{t})$ define the carrier signal and the modulating signal, respectively. Here, the offset is $B=2$ and must be subtracted from the results of Hilbert transformation to obtain the modulating signal. Of course, when working with data obtained in experimental or numerical studies, the analytical form of the carrier and modulating signals that form the raw signal $x(t)$ is unknown.

If we apply Hilbert transformation to the signal $x(t)$ [Fig. 1(a)], as described above, we can find the modulating wave $m(t)$, which is given as the red line in Fig. 1(b).

\section{DECOUPLING BY FILTERING AND HILBERT TRANSFORMATION}

Figure 2 shows the process for amplitude-modulation analysis following Mathis et $a .^{5}$ In Sec. III, we generated a
FIG. 2. Decoupling procedure ${ }^{5}$ for twopoint analysis. 
sinusoidal signal corresponding to the smaller scale in Fig. 2 to describe how to obtain the envelope by Hilbert transformation. The correlation coefficient $\mathrm{R}$ can be obtained by using either the modulating wave or its filtered envelope depending on the complexity of the raw signal.

Figure 2 shows a two-point application in which the larger scale (LS) is obtained from the instantaneous streamwise fluctuating velocity signal in the log-law region and the smaller scale (SS) is obtained at the location of the inner peak of the kinetic energy $k\left(y^{+} \simeq 15\right)$. Amplitude-modulation analysis is also possible with one-point application, in which both the SS and LS signals are obtained from the inner velocity signal (see Sec. VI A 2).

The subscripts $i, o, S$, and L correspond to "inner," "outer," "small-scale," and "large-scale," respectively. For instance, $u_{i, \mathrm{~S}}^{+}$ and $u_{i, L}^{+}$represent the small and large scales, respectively, at the inner peak location. For the outer peak location, $u_{0, S}^{+}$and $u_{0, L}^{+}$represent small and large scales, respectively.

\section{SCALE DECOMPOSITION BY EMPIRICAL MODE DECOMPOSITION BY HILBERT-HUANG TRANSFORMATION}

EMD by Hilbert-Huang transformation is based on the assumption that any data signal consists of different simple intrinsic modes of oscillations, the raw signal being a superposition of those oscillations. Each oscillatory mode is referred to as an IMF ${ }^{11}$ and satisfies the following two conditions: (i) the numbers of local extrema and zero crossings must be equal or differ by one at the most, and (ii) the mean value of the curve constructed by connecting the maxima and minima should always be zero.

\section{A. EMD algorithm}

Let $X(t)$ be a continuous time series to which we apply EMD by means of the following algorithm.

i. Find all the local maxima and local minima; see Fig. 3(a).

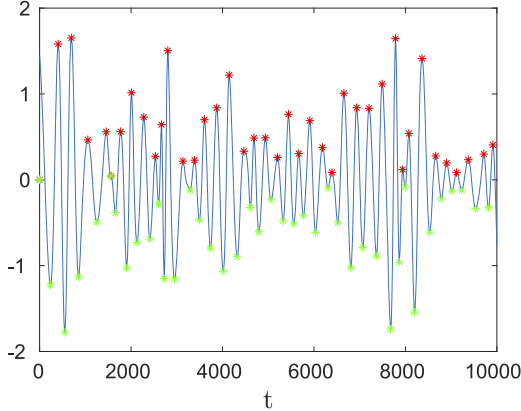

(a)

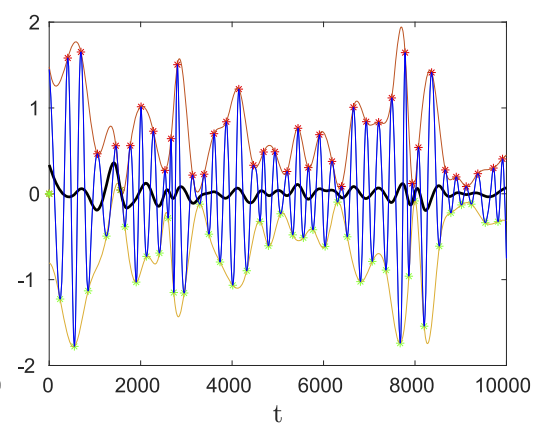

(b)
FIG. 3. Illustration of procedure for finding local maxima and applying a cubic spline. (a) All local maxima (red points) and local minima (green points). (b) Apply a cubic spline and construct the mean curve ( $m_{11}$, the thick black line).
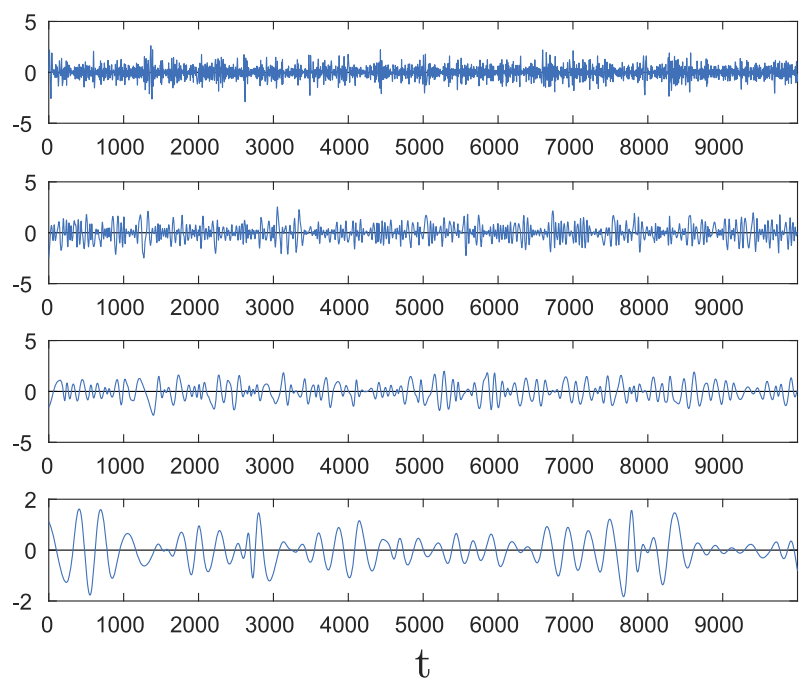

(a)
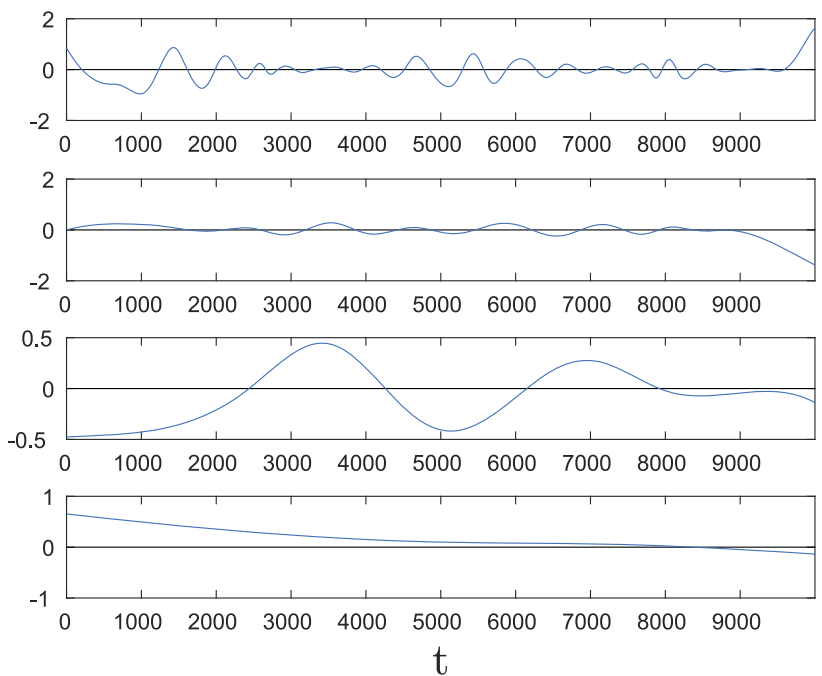

(b)

FIG. 4. An example of obtained intrinsic mode functions (IMFs) by applying empirical mode decomposition (EMD). The upper signal in the left figure is the raw data, and the subsequent seven signals are the IMFs obtained by applying EMD to the raw data. (a) The raw data (most upper signal) and the first three IMFs. (b) IMFs 4-6 and the residual. 


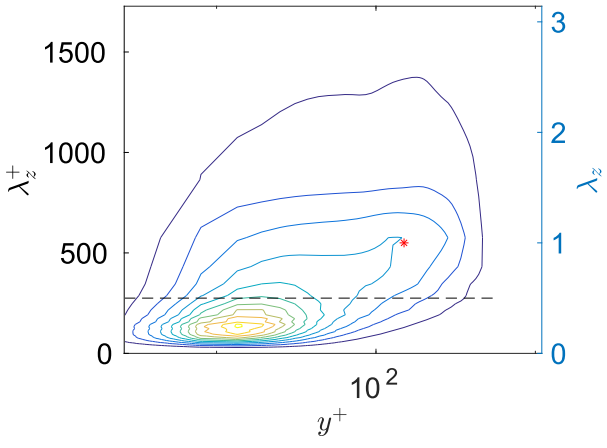

FIG. 5. Contours of pre-multiplied energy spectra $k_{z} \phi_{u u}\left(k_{z}\right) / u_{\tau}^{2}$ for streamwise velocity fluctuations. The "outer peak" is indicated by the red star, and the dashed line marks the cutoff wavelength.

ii. Construct envelope curves for the maxima and minima and obtain the mean curve of these two envelope curves, i.e., $m_{11}(\mathrm{t})$; see Fig. $3(\mathrm{~b})$.

iii. Calculate $h_{11}(t)=h_{10}(t)-m_{11}(t)$. The first IMF is constructed from the raw data, i.e., $h_{10}=X(t)$. The first index in $h_{i j}$ represents the number of the IMF in the construction, and the second represents the iteration number.

iv. Steps (i)-(iii) are performed recursively: $h_{1 k}(t)=h_{1(k-1)}(t)$ $-m_{1 k}(t)$. The stopping criterion is defined as follows: let $0 \leq \mathrm{t} \leq \mathrm{T}$, then

$$
s d_{n}=\sum_{t=0}^{T}\left(\frac{\left|h_{n(k-1)}(t)-h_{n k}(t)\right|^{2}}{h_{n(k-1)}^{2}(t)}\right) .
$$

Empirically, a number $s d_{n}<\epsilon$ is used as the stopping criterion, where $\epsilon$ is between 0.2 and 0.3 .

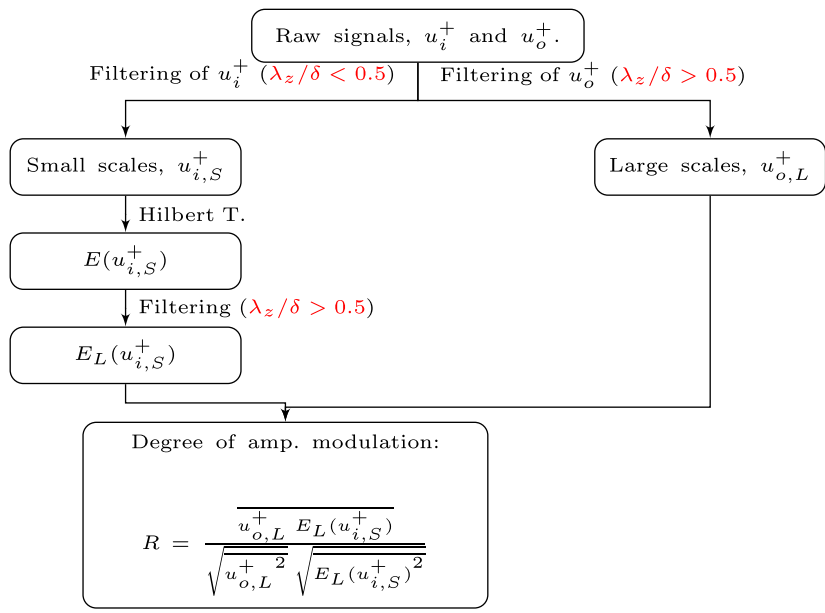

(a) v. When the first IMF $h_{1 k}(t)$ is found, it is subtracted from $h_{10}(t)$ to give $h_{20}(t)$. The process then restarts from step (i) to find the second IMF.

vi. Set $c_{i}(t)=h_{i k}(t)$, where $c_{i}(t)$ is the $i$ th IMF. The overall calculation stops when subtraction at step (v) gives either monotonic or constant data.

Consequently, a set of IMFs is obtained (Fig. 4).

\section{SCALE DECOMPOSITION BASED ON FILTERINC VERSUS EMD}

To define the energy map, the pre-multiplied energy spectra for the streamwise velocity fluctuations are plotted. The energy peak located in the near-wall region is called the "inner peak" and is usually regarded as the energetic signature of the near-wall cycle of turbulence production. ${ }^{5,18}$ In Fig. 5, this inner peak is located near $y^{+}=15$ and $\lambda_{z}^{+}=100$. The socalled "outer peak" is located in the logarithmic region and is usually seen as the energetic signature of the large-scale organization of the velocity field (superstructures). In Fig. 5, this outer peak is located near $y^{+}=150$ and $\lambda_{z}=1$.

According to the energy map, $\lambda_{z} / \delta=0.5$ could be a reasonable cutoff wavelength for the present study. First, the filtering-based method shown in Fig. 6(a) is used to investigate the amplitude modulation effect of the outer LS structures on the inner SS structures. ${ }^{5}$ The outer data $u_{0}^{+}\left(\right.$from $y^{+}=150$ ) enter the process shown in Fig. 6(a) from the right-hand side, while the inner data $u_{i}^{+}\left(\right.$from $\left.y^{+}=15\right)$ enter from the left-hand side.

By applying a low-pass filter to the outer fluctuation data $u_{0}^{+}$, we obtain the large scales $u_{o, L}^{+}$. By applying a high-pass filter to the inner data $u_{i}^{+}$, we obtain the small scales $u_{i, \mathrm{~S}}^{+}$. Then, by applying Hilbert transformation to $u_{i, S}^{+}$, we obtain an envelope $\mathrm{E}\left(u_{i, \mathrm{~S}}^{+}\right)$for the inner SS data. According to Mathis et al., ${ }^{5}$ this

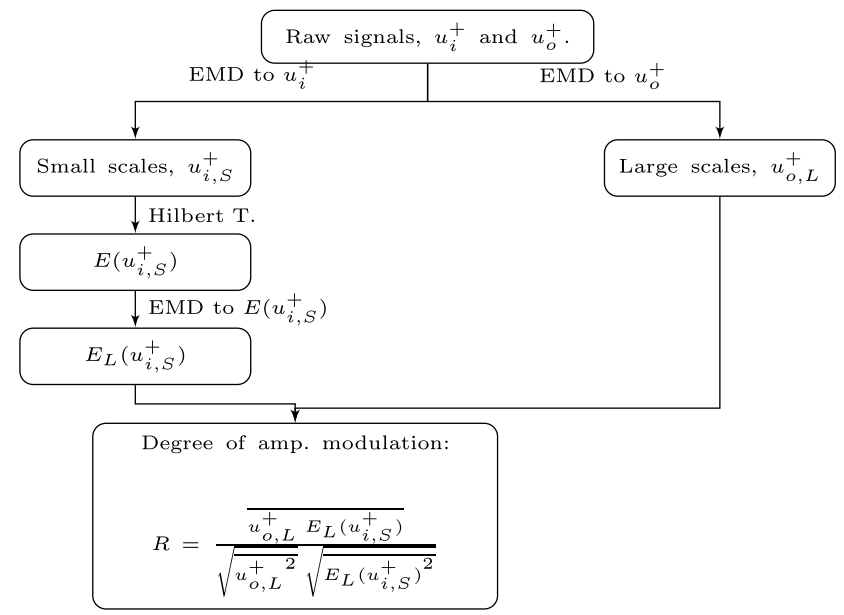

(b)

FIG. 6. Illustration of decoupling procedures of the two methods. $u_{i}^{+}$is from $y^{+}=15$, and $u_{0}^{+}$is from $y^{+}=150$. (a) Decoupling procedure based on filtering. ${ }^{5}$ (b) Proposed decoupling procedure based on EMD. 


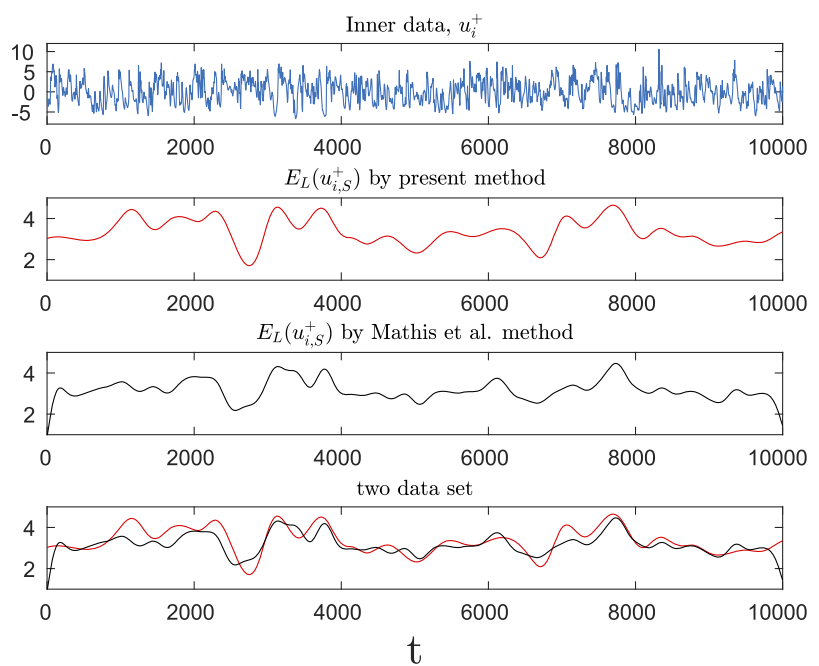

(a)

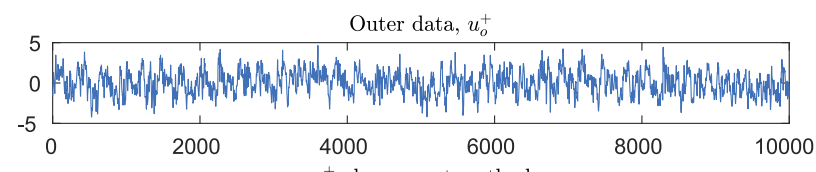

$u_{o, L}^{+}$by present method
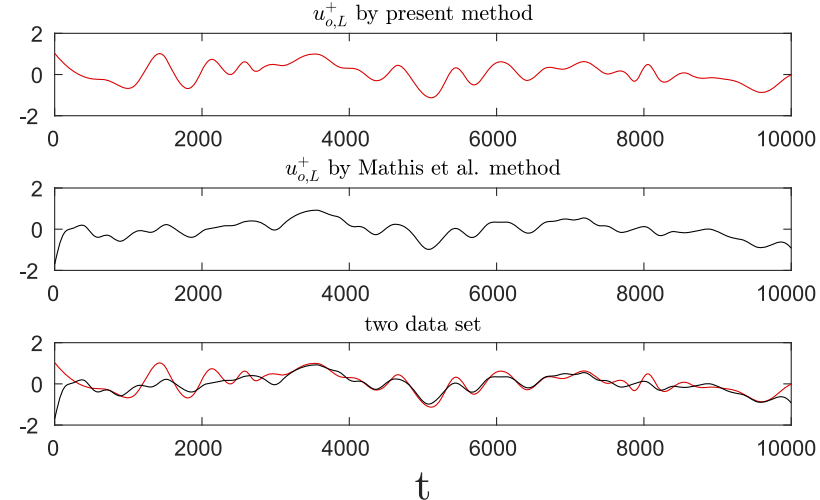

(b)

FIG. 7. Results of applying the right- and left-hand side versions of the two methods [see Figs. $6(\mathrm{a})$ and $6(\mathrm{~b})$ ]. Red line: present method; black line: method of Mathis et al.5 (a) Left-hand side version. (b) Right-hand side version.
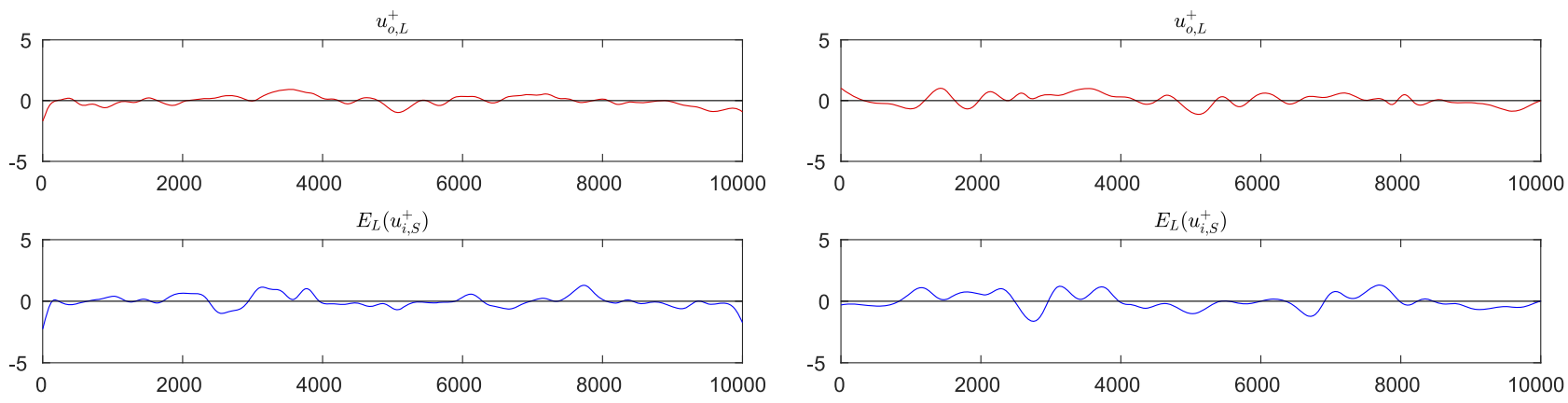

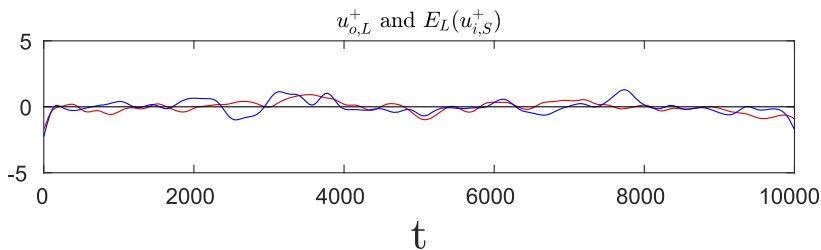

(a)

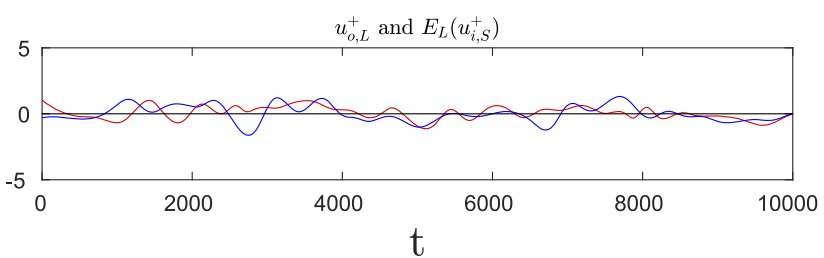

(b)

FIG. 8. $u_{\mathrm{o}, \mathrm{L}}^{+}$and $\mathrm{E}_{\mathrm{L}}\left(u_{i, \mathrm{~S}}^{+}\right)$for conventional and EMD-based approximations, the latter being the present method: two-point analysis. (a) Two-point analysis by filtering. ${ }^{5}$ (b) Two-point analysis by EMD-based procedure.

\begin{tabular}{|c|c|c|c|}
$\begin{array}{c}\text { Large scale } \\
\text { (outer) }\end{array}$ & Footprinting & $\begin{array}{c}\text { Large scale } \\
\text { (inner) }\end{array}$ & Small scale \\
Modulation effect & Modulation effect
\end{tabular}

FIG. 9. Modulation effect can be analyzed using either outer or inner largescale (LS) data. 

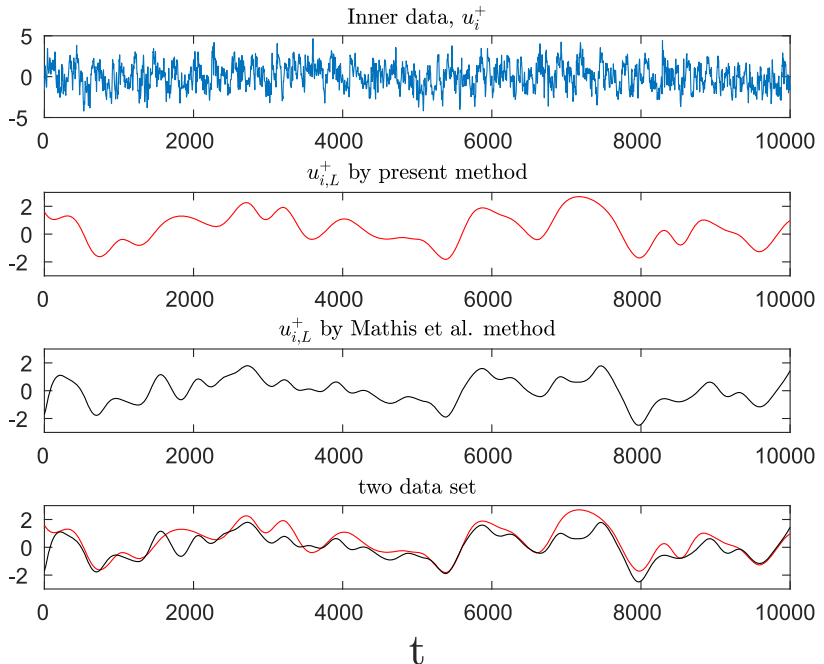

FIG. 10. Results obtained with the right-hand side version of the two methods [see Figs. 6(a) and 6(b)]: one-point analysis. Red line: present method; black line: method of Mathis et al.

envelope tracks not only the LS modulation due to the outer LS structures but also the SS structures of the inner data. Therefore, to remove this effect, we must low-pass filter the envelope to obtain the data $E_{L}\left(u_{i, S}^{+}\right)$.

Here we suggest using the process shown in Fig. 6(b) to investigate the amplitude modulation for a given signal based on EMD. In the present study, the first four IMFs are summed to obtain the SS data and the fifth IMF is taken as the LS data. $u_{O, L}^{+}$is obtained by applying EMD to the outer data [right-hand side of Fig. 6(b)]. On the left-hand hand side, in the first step, EMD is applied to the inner data $u_{i}^{+}$, and the first four IMFs are summed to obtain the SS $u_{i, S}^{+}$. Hilbert transformation is then applied to obtain the envelope $\mathrm{E}\left(u_{i, \mathrm{~S}}^{+}\right)$of the SS data. Finally, EMD is applied again and the fifth mode is taken as $E_{L}\left(u_{i, S}^{+}\right)$to extract the modulation effect on the SS structures of the inner data.

\section{A. Results and discussion}

\section{Two-point analysis}

The data $E_{L}\left(u_{i, S}^{+}\right)$obtained with the left-hand side method [see Figs. 6(a) and 6(b)] match very well [Fig. 7(a)]. The LS data obtained by low-pass filtering (black line) and the fifth IMF mode obtained by EMD also match almost perfectly [Fig. 7(b)].

Figures 7(a) and 7(b) show that the present method is compatible with that of Mathis et al. ${ }^{5}$ However, the main aim of analyzing the modulation effect is to find the correlation between the LS and SS data. In Figs. 8(a) and 8(b), the LS data, $u_{o, L}^{+}$, of the outer logarithmic region and $E_{L}\left(u_{i, S}^{+}\right)$of the inner region are plotted for the present method and that of Mathis et al. ${ }^{5}$ Both methods exhibit correlation for negative fluctuations in the same time intervals, namely, between 4000 and 6000 and after 8000. For the correlation coefficient $R$ that defines the degree of modulation, we obtain values of 0.26 and 0.29 with the method of Mathis et al. ${ }^{5}$ and the present method, respectively.

\section{One-point analysis}

The one-point analysis to estimate the degree of amplitude modulation should give similar results to those of the two-point analysis. This is based on the idea that the LS
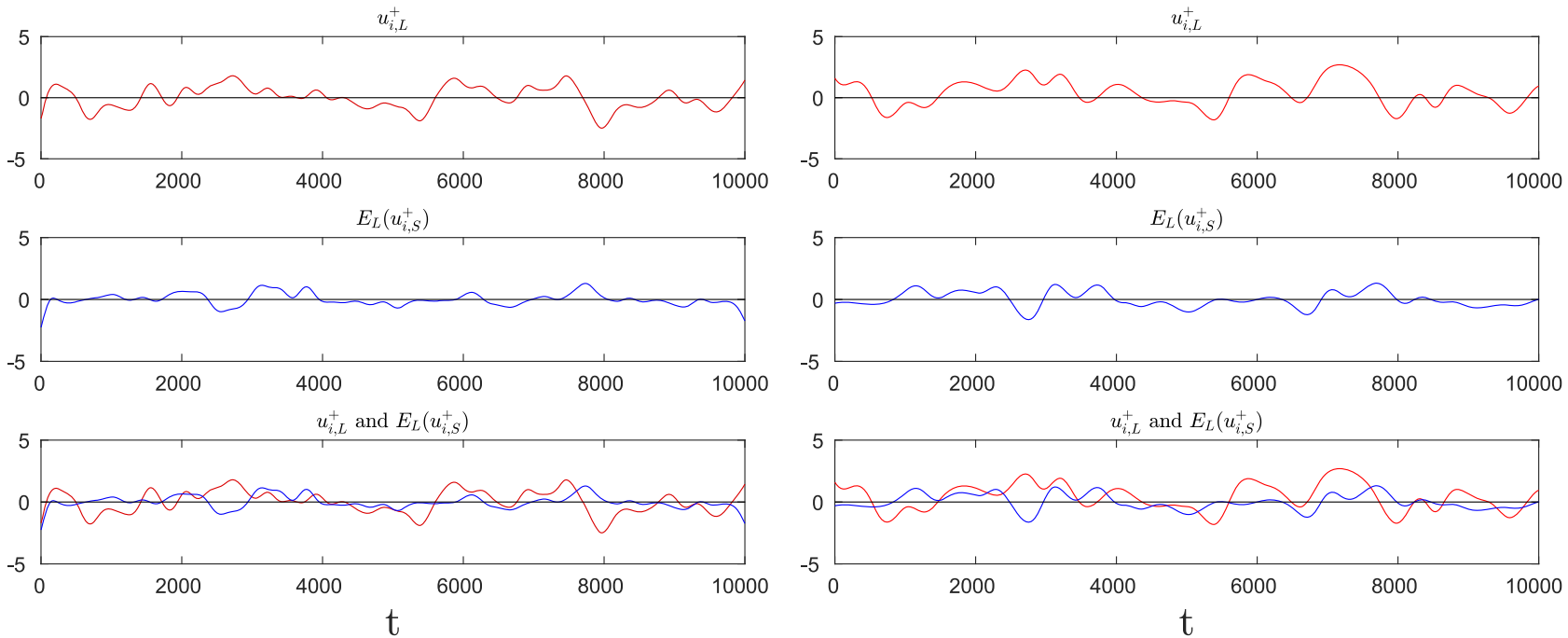

(a)

(b)

FIG. 11. $u_{i, L}^{+}$and $E_{L}\left(u_{i, S}^{+}\right)$for both conventional and EMD-based approximations: one-point analysis. (a) One-point analysis with the filtering method of Mathis et al. ${ }^{5}$ (b) One-point analysis with EMD-based procedure. 
_ original signal

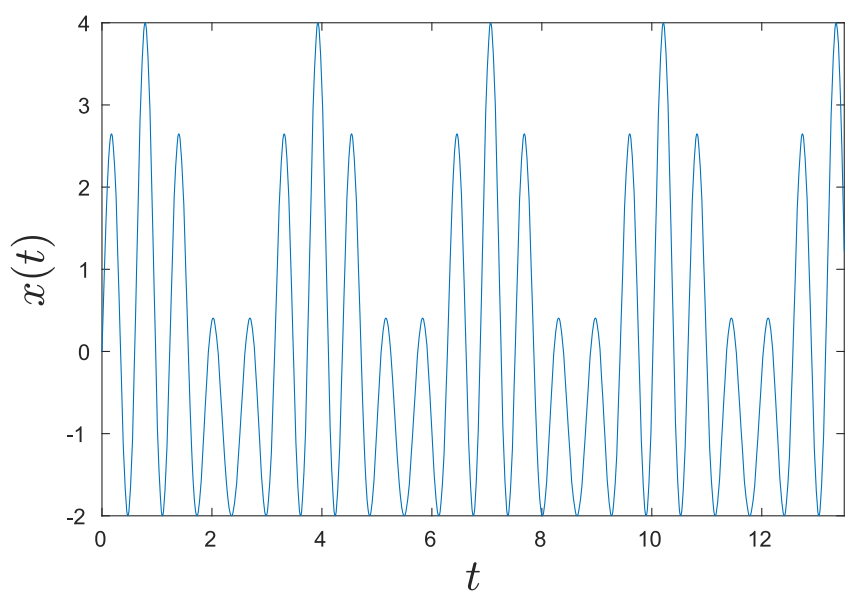

FIG. 12. Raw signal $x(t)=(2+\sin (2 t)) \sin (10 t)+\sin (2 t)$.

motions from the outer layer affect the SS inner structures via footprinting on the LS inner structures, a relationship that is illustrated in Fig. 9.

Therefore, in this part, we consider data obtained at $y^{+}=15$. The same procedure is applied as using the two-point analysis given in Figs. 6(a) and 6(b) to decompose the signal into its LS and SS components, represented as $u_{i, L}^{+}$and $u_{i, S}^{+}$, respectively. The LS data obtained by low-pass filtering (black line) and the fifth IMF mode obtained by EMD are shown, and they match almost perfectly (Fig. 10), similar to two-point analysis results. Only the right-hand side version of the methods is used because the left-hand side version is the same as in Fig. 7(a) that is obtained from the inner data.

In Figs. 11(a) and 11(b), the LS data $u_{i, L}^{+}$of the inner logregion and $E_{L}\left(u_{i, S}^{+}\right)$of the inner region are plotted for the method of Mathis et al. ${ }^{5}$ and the present method. Both methods exhibit correlation for negative fluctuations at the same time intervals of 4000-6000 and after 8000, similar to the two-point analysis, as expected. With the method of Mathis et $a l .5$ and the present method, we obtain values of 0.01 and 0.12 , respectively, for the correlation coefficient $R$ that defines the degree of modulation.

It is very interesting that there is a huge difference in the correlation coefficient $\mathrm{R}$ despite the similarity between the LS data obtained by filtering and EMD (Fig. 10). For the present method, comparing the one-point and two-point analyses, a slightly lower correlation coefficient is reasonable because the modulation effect is obtained indirectly by the footprint of the superstructures for the former (Fig. 9). Therefore, the correlation coefficient $R=0.12$ seems reasonable for the one-point analysis compared to $R=0.29$ obtained for the two point analysis with the EMD-based method.

\section{Validation of correlation coefficient $R$ with simple sinusoidal signal}

We now add an LS part to the equation used in Sec. III, which is equal to the modulating signal $m(t)=\sin (2 t)$, namely,

$$
x(t)=(B+\sin (2 t)) \sin (10 t)+\sin (2 t) .
$$

In Sec. III, we used the SS part of this signal, namely, $(2+$ $\sin (2 t)) \sin (10 t)$, to explain the amplitude modulation. Now we
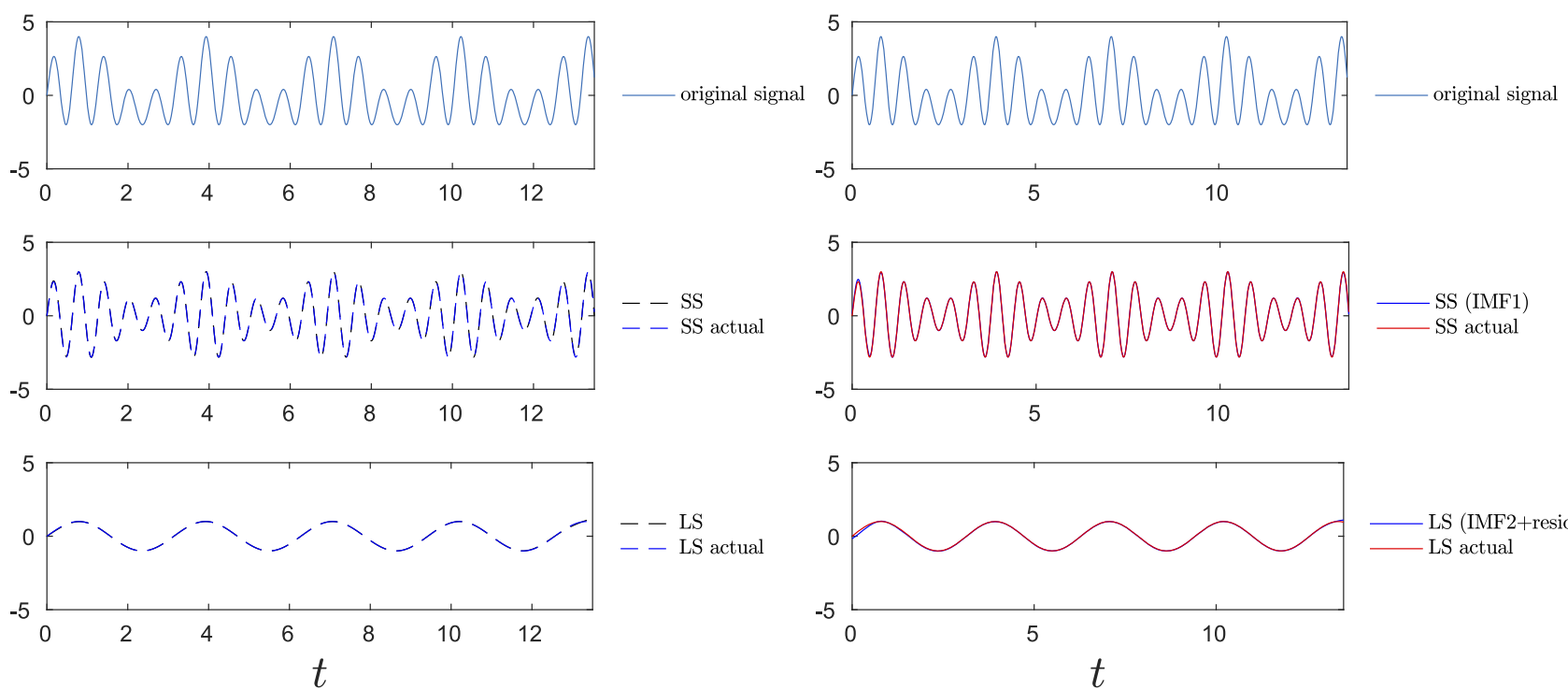

(a)

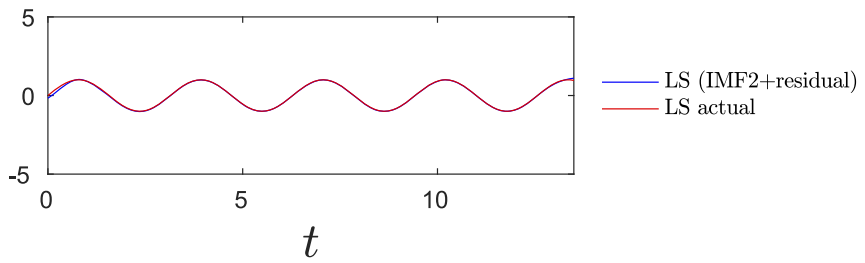

(b)

FIG. 13. Validation of conventional and EMD-based decoupling procedures with a simple sinusoidal signal. (a) Analysis results by filtering. ${ }^{5}$ (b) Analysis results by EMD-based decoupling procedure. 
add $\sin (2 t)$ to the signal, which corresponds to the LS part of the signal $x(t)$. By decomposing the signal with the EMD and filtering methods, we separate the LS data as $x_{\mathrm{LS}}$ and the SS data as $x_{S S}$. We then apply Hilbert transformation to the SS part and obtain an SS envelope $E_{L}\left(x_{S S}\right)$. Note that with the existing method, no second filtering is necessary because of the relative simplicity of the raw signal $x(t)$. After those operations, both $E_{L}\left(x_{S S}\right)$ and $x_{L S}$ should be very close to the signal $\sin (2 t)$. The error, namely, the difference between the LS and SS envelopes obtained and the actual signals [which are both $\sin (2 t)]$, is found to be less than 0.05 for both methods.

The original signal is shown in Fig. 12. Both methods clearly divide the original signal into LS and SS parts and successfully define an envelope to the SS part by Hilbert transformation [Figs. 13(a) and 13(b)]. The correlation coefficient is $\mathrm{R}=0.99$ in both cases, thereby showing that both methods have identical reference for relatively simple data. However, with more-complicated data, the decomposition method could be an important factor.

\section{CONCLUSION}

In this study, an EMD-based decoupling procedure is suggested and compared with the existing filteringbased approximation for amplitude-modulation analysis. It is shown that the LS and SS signals obtained with either filtering or EMD follow almost the same path and capture the correlation for negative fluctuations, with the same time intervals. However, although the signals obtained with EMD and filtering-based amplitude-modulation analysis approximations exhibit qualitatively very similar signals, quantitative analysis by comparing the correlation coefficient $R$, which defines the degree of modulation, may vary. Comparable correlation values are obtained in two-point analysis, namely, $R$ $=0.26$ and 0.29 for the filtering and EMD cases, respectively. While a reduction is expected for the one-point analysis compared to the two-point analysis because of the indirect effect of footprinting, the filtering-based approximation gives too low a correlation coefficient $\mathrm{R}$ for the one-point analysis compared with the two-point analysis, namely, $R=0.01$. However, the EMD-based approximation gives a correlation coefficient of $\mathrm{R}=0.12$ for the one-point analysis, which is a reasonable value compared to that obtained with two-point analysis. Therefore, we claim that signals decoupled to large and small scales by EMD-based amplitude analysis better capture the correlation compared to the conventional filtering-based amplitude-analysis approximation.

\section{ACKNOWLEDGMENTS}

This work was supported by the European Commission through the Research and Innovation action DRAGY (Grant
Agreement No. 690623) and by the Ministry of Industry and Information Technology (MIIT) of the Chinese government.

\section{REFERENCES}

${ }^{1} \mathrm{~J}$. Jiménez, "The largest scales of turbulent wall flows," in Center for Turbulence Research Annual Research Briefs (Stanford University, 1998).

${ }^{2}$ R. J. Adrian, C. D. Meinhart, and C. D. Tomkins, "Vortex organization in the outer region of the turbulent boundary layer," J. Fluid Mech. 422, 1-54 (2000).

${ }^{3}$ J. Jiménez, J. C. Del Alamo, and O. Flores, "The large-scale dynamics of near-wall turbulence," J. Fluid Mech. 505, 179-199 (2004).

${ }^{4} \mathrm{~N}$. Hutchins and I. Marusic, "Evidence of very long meandering features in the logarithmic region of turbulent boundary layers," J. Fluid Mech. 579, 1-28 (2007).

${ }^{5}$ R. Mathis, N. Hutchins, and I. Marusic, "Large-scale amplitude modulation of the small-scale structures in turbulent boundary layers," J. Fluid Mech. 628, 311-337 (2009).

${ }^{6} \mathrm{P}$. Schlatter and R. Örlü, "Quantifying the interaction between large and small scales in wall-bounded turbulent flows: A note of caution," Phys. Fluids 22, 051704 (2010).

${ }^{7}$ R. Mathis, I. Marusic, N. Hutchins, and K. Sreenivasan, "The relationship between the velocity skewness and the amplitude modulation of the small scale by the large scale in turbulent boundary layers," Phys. Fluids 23, 121702 (2011).

${ }^{8}$ B. Ganapathisubramani, N. Hutchins, J. Monty, D. Chung, and I. Marusic, "Amplitude and frequency modulation in wall turbulence," J. Fluid Mech. 712, 61-91 (2012).

${ }^{9} \mathrm{~L}$. Agostini and M. Leschziner, "On the influence of outer large-scale structures on near-wall turbulence in channel flow," Phys. Fluids 26, 075107 (2014).

${ }^{10}$ L. Agostini, M. Leschziner, and D. Gaitonde, "Skewness-induced asymmetric modulation of small-scale turbulence by large-scale structures," Phys. Fluids 28, 015110 (2016).

${ }^{11}$ N. E. Huang, "Introduction to the Hilbert-Huang transform and its related mathematical problems," in Hilbert-Huang Transform and its Applications, edited by N. E. Huang and S. S. P. Shen (World Scientific, 2005), pp. $1-26$.

${ }^{12}$ P. J. Schmid, "Dynamic mode decomposition of numerical and experimental data," J. Fluid Mech. 656, 5-28 (2010).

${ }^{13}$ S. Le Clainche and J. M. Vega, "Higher order dynamic mode decomposition to identify and extrapolate flow patterns," Phys. Fluids 29, 084102 (2017).

${ }^{14}$ L. Davidson and S.-H. Peng, "Hybrid LES-RANS: A one-equation SGS model combined with a $k-w$ model for predicting recirculating flows," Int. J. Numer. Methods Fluids 43, 1003-1018 (2003).

${ }^{15} \mathrm{H}$. Nilsson, "A parallel multiblock extension to the CALC-BFC code using PVM," Technical Report 9711, Department of Thermo and Fluid Dynamics, Chalmers University of Technology, Gothenburg, 1984.

${ }^{16}$ P. Emvin and L. Davidson, "Development and implementation of a fast large eddy simulations method," Department of Thermo and Fluid Dynamics, Chalmers University of Technology, Gothenburg, 1997, http://www.tfd. chalmers.se/ lada/postscript_files/emvin_phd_paper_V.pdf.

${ }^{17}$ C. Rhie and W. L. Chow, "Numerical study of the turbulent flow past an airfoil with trailing edge separation," AIAA J. 21, 1525-1532 (1983).

${ }^{18} \mathrm{M}$. Bernardini and S. Pirozzoli, "Inner/outer layer interactions in turbulent boundary layers: A refined measure for the large-scale amplitude modulation mechanism," Phys. Fluids 23, 061701 (2011). 